\title{
DINÁMICA DE LA INVERSIÓN EN LA ECONOMÍA ESPAÑOLA (1982-2007): EXPECTATIVAS, DEMANDA Y FINANCIACIÓN
}

\author{
LUIS CÁRDENAS DEL REY \\ Departamento de Administración y dirección de empresas, UNIVERSIDAD ISABEL I, ESPAÑA \\ E-mail: luiscard@ucm.es
}

\section{RESUMEN}

El objeto principal de este trabajo es analizar la dinámica inversora que ha mostrado la economía española durante el período expansivo 1982-2007, caracterizado por el fuerte aumento del sector de la construcción, con el objetivo de explicar las causas que determinaron un patrón de crecimiento muy dinámico. Combinando distintos elementos teóricos de las principales hipótesis sobre los determinantes de la inversión, se propone un marco que permite analizar simultáneamente los tres tipos de agentes inversores (hogares, Estado y empresas), basado en las categorías de expectativas, demanda y financiación. Utilizando los datos de la base BBVA-IVIE y una metodología de series temporales obtenemos que la inversión efectuada por los distintos agentes muestra una serie de interdependencias que dan como resultado el papel predominante que las "actividades vinculadas con la construcción" tienen como motor de la inversión en su conjunto.

Palabras clave: Inversión, crecimiento, ciclos económicos, financiación, economía española

\section{Investment growth in the Spanish economy (1982-2007): expectations, demand and finance}

\begin{abstract}
This paper studies one of the most paradigmatic facts of Spanish economic growth during the period 1982-2007: high growth of all types of investment. The primary purpose is to explain the causal factors of its growth pattern. We build an analytical framework that combines the investment theories; allowing to analyze simultaneously the investment of the three types of agents (households, Government and corporations), with three categories: expectations, demand and financing. Using the BBVA-IVIE database and a time series approach, our findings indicate that "activities connected to construction" are of primary importance in the investment pattern. This fact is interpreted as the result of the interdependencies between agents.
\end{abstract}

Key Words: Investment, demand, expectations, financing, Spanish economy

Thematic Area: Applied Economics and Methods

Clasificación JEL: E20, E22, E32 


\section{INTRODUCCIÓN}

En este trabajo se analiza la dinámica inversora en la economía española durante el período 19822007, que presenta la gran particularidad del fuerte auge del sector de la construcción, con el objetivo de explicar las causas que determinaron su crecimiento y localización sectorial.

En primer lugar, se presentan las aportaciones realizadas por distintas propuestas teóricas basadas en un enfoque de demanda, acerca de cuáles son los principales factores que explican el comportamiento de la inversión. En segundo lugar, se sintetiza un conjunto de hechos observados en la economía española referidos a la evolución de la inversión y de las principales variables que esas propuestas teóricas proponen como factores explicativos. A partir de estos elementos teóricos y de estas evidencias empíricas, se formulan dos hipótesis que pretenden combinar el análisis desagregado de la inversión efectuada por los distintos agentes (hogares, Estado y empresas) con el análisis agregado de la inversión efectuada por el conjunto de la economía.

Esta metodología de análisis ofrece dos ventajas interesantes. De un lado, la mayor parte de la literatura sobre la inversión se centra en el comportamiento de las empresas como si fuera similar al de toda la economía. Sin embargo, los hechos muestran que, como promedio del período, más del $40 \%$ de la formación bruta de capital fijo corresponde a la inversión residencial de los hogares y a la inversión, sobre todo en infraestructuras, de las administraciones públicas; siendo dudoso que las decisiones de los hogares y el Estado estén motivadas por los mismos factores que la inversión empresarial. De otro lado, esta metodología abre la posibilidad de que exista una vinculación entre las inversiones realizadas por los tres agentes, de manera que el comportamiento agregado en el conjunto de la economía puede requerir la incorporación de elementos explicativos que no están presentes en el análisis desagregado.

El documento está estructurado en cinco apartados que siguen a este de introducción. El segundo presenta los fundamentos teóricos que desde un enfoque de demanda se consideran como factores determinantes de la inversión. El tercero expone los hechos observados en la economía española de 1982-2007 y formula las dos hipótesis a investigar. Los dos apartados siguientes indagan en esas hipótesis utilizando varios modelos econométricos. Finalmente, el sexto apartado muestra las conclusiones del análisis realizado.

\section{FUNDAMENTOS TEÓRICOS}

\subsection{Principales elementos destacados por la literatura}

La literatura de referencia sobre los determinantes de la inversión proporciona varios factores explicativos (Cárdenas, 2018). En primer término, desde el lado de la demanda destacan dos factores básicos: i) las expectativas de rentabilidad que tienen los empresarios y ii) el grado de utilización de la capacidad productiva instalada. Sin embargo, existen diferencias entre ellas cuando tratan de precisar cuáles son las variables en las que se concretan ambos factores.

Por un lado, se proponen distintas variables para concretar las expectativas de rentabilidad que, por definición, no son conocidas en la medida en que se refieren a un futuro radicalmente incierto en el sentido keynesiano. Unos autores proponen la tasa de ganancia, que relaciona el nivel de beneficio con el capital instalado $(r=B / K)$, pero al tratarse de una tasa esperada caben diferentes especificaciones sobre el modo de convertir la tasa contemporánea (r) en tasa esperada (re), bien sea considerando el tipo de interés (nominal o real), o bien la tasa de ganancia tendencial de un período u otras variantes (Allsopp y Glyn, 1999; Richardson y Romilly, 2008). En ese sentido, Glyn (1997) recopila un conjunto de alternativas presentes en la literatura para señalar las dificultades técnicas que plantea la obtención de buenas estimaciones de la tasa de beneficio. Otros autores utilizan directamente la variable de los beneficios como referencia (Sherman, 2014), mientras que otros siguen la propuesta de Weisskopf (1979), después popularizada por los modelos que utiliza como indicador la cuota del beneficio en la renta (B/Y), a la que identifican con el grado de monopolio siguiendo a Steindl (1952).

Por otro lado, el grado de utilización de la capacidad instalada se define por la relación entre la producción efectiva y la potencial $\left(\mathrm{u}=\mathrm{Y} / \mathrm{Y}^{*}\right)$, o bien entre el capital utilizado y el instalado $(\mathrm{u}=$ 
$\mathrm{Ki} / \mathrm{K})$. Con frecuencia, ese factor se identifica con el nivel de demanda efectiva, por lo que muchos autores utilizan directamente esta última variable $(\mathrm{Y})$, con un planteamiento similar al propuesto por la teoría del acelerador. Otros autores lo identifican con la eficiencia del capital instalado ( $\mathrm{Y} / \mathrm{K})$, de modo que al utilizar la cuota de beneficio $(\mathrm{B} / \mathrm{Y})$ combinando ambas ratios obtienen la tasa de rentabilidad o ganancia $(\mathrm{B} / \mathrm{K})$.

Recogiendo ambas propuestas, Blecker (2015) formula que el efecto acelerador predomina sobre la inversión en el largo plazo, mientras que los beneficios ejercen su influencia a corto plazo. Sin embargo, otros autores se inclinan por emplear como variable el grado de utilización que se elabora a través de encuestas empresariales, si bien su información se refiere a las empresas del sector industrial incluyendo a veces al sector de la construcción.

En segundo término, junto con las expectativas empresariales y la utilización de capacidad o nivel de demanda, un tercer factor que la literatura señala como determinante de la inversión es el que concierne a las condiciones monetario-financieras. Aunque cuenten con ahorro propio (generado por los beneficios no distribuidos), las empresas financian parte de su inversión con recursos ajenos, que provienen de préstamos bancarios y/o de bonos emitidos en los mercados de capital. Incluso bajo el supuesto de que la oferta de dinero sea elástica a la demanda de financiación de las empresas, derivada de las decisiones de inversión. No obstante, las empresas están sometidas al principio kaleckiano del "riesgo creciente" (Kalecki, 1937), en la medida en que asumen compromisos fijos de devolución de sus deudas a partir de flujos de ingresos esperados e inestables. En otras palabras, la inversión financiada con esos recursos (bienes de capital) requiere de plazos más largos (para producir y vender) y los ingresos dependen de las condiciones de la demanda, mientras que los pagos (amortización e intereses) están previamente fijados.

En tercer lugar, las condiciones financieras pueden aproximarse mediante distintas variables, según que se refieran sólo al mercado de dinero (préstamos) o también al mercado de capitales (bonos, acciones). Cabe, pues, utilizar indicadores relativos a los tipos de interés nominales y reales (Seguino, 1999; Epstein, 1994), a las cotizaciones bursátiles o al nivel de endeudamiento de las empresas (Hein, 2014). Además, esos indicadores pueden tomarse en sentido positivo, como factores que facilitan la financiación de la inversión, o bien, en sentido negativo, como factores restrictivos si el endeudamiento resulta excesivo y genera amenazas de fragilidad financiera, debidas al encarecimiento y/o los recortes de la oferta de crédito o a la posibilidad de impago por parte de las empresas endeudadas (Courvisanos, 1995; Mott, 2009).

Por último, pueden considerarse otros factores que afectan a las decisiones de inversión, tales como los alicientes creados en períodos de intenso desarrollo tecnológico, las consecuencias del contexto social y político, el funcionamiento de las instituciones, la tradición cultural de las empresas y otros (Crotty, 1992; Courvisanos, 2012).

\subsection{Análisis de la función de inversión en España}

Lo cierto es que la evidencia sobre la función de inversión en España es desigual. Andrés et al. (1990) acercan el coste de uso del capital y el "acelerador flexible" al grado de utilización de la capacidad; Estrada et al. (1997) incorporan los factores financieros con variables que se aproximan a la interpretación de Fazzari y Mott (1987) aplicando el principio de riesgo creciente; mientras que Azofra y López (1997) y Moral (2008) trabajan con la teoría de los cash-flows desde la perspectiva macroeconómica y la teoría pecking order desde la perspectiva de las empresas.

Otros autores interpretan la "Q de Tobin" de modo que establecen una relación entre la inversión y una tasa de rentabilidad que descuenta el tipo de interés (profitability gap), tanto para estudios de la economía española en su conjunto (Blanch, 1988; Espasa y Senra, 1993; Esteve y Tamarit, 1994), como para análisis más específicos sobre las manufacturas (Alonso y Bentolila, 1994). El trabajo de Posada, Urtasun y González (2014) combina ambos enfoques estimando un modelo de corrección de error para el período 1995-2013, en el que encuentran que a largo plazo destacan tres factores: la producción agregada, la ratio entre la remuneración por asalariado y el coste de uso del capital, y la Q de Tobin; mientras que, a corto plazo, destacan cinco variables: la demanda agregada, el grado de 
utilización, la posición financiera de las empresas, los márgenes comerciales y una proxy referida a la incertidumbre.

Por su parte, desde enfoques de demanda, la literatura disponible es más reducida y puede clasificarse según el énfasis en los factores considerados como determinantes de la inversión. En primer término, Arriola (1999) plantea una función de inversión como la de Steindl, aunque realmente se centra en aspectos cualitativos relacionados con el grado de utilización y su vinculación con la demanda agregada en el caso de la economía vasca. Su principal resultado es que la subutilización de capacidad instalada se produce por falta de demanda efectiva. En un estudio más amplio, Muñoz de Bustillo y Fernández (2007) desagregan la rentabilidad en términos de beneficios y utilización, de forma que un mayor nivel de capacidad utilizada se plasma en una mayor rentabilidad. A partir de la base EUCOWE encuentran que la economía española presenta menores tasas de utilización de la capacidad productiva que otros países europeos y que las mayores ratios corresponden a las grandes empresas y a otras que mantienen una mayor continuidad en su demanda.

Felipe (2002) propone una función de inversión para la economía española que depende de los dos elementos canónicos: la rentabilidad y la utilización de capacidad, y los relaciona con la evolución del desempleo. Encuentra que existe una relación negativa entre las dos variables explicativas (rentabilidad y utilización) con respecto al desempleo. Por su parte, Arestis, González y Dejuán (2012) aplican una función de inversión de inspiración kaleckiana en el que incorporan como variables explicativas: la demanda agregada, los tipos de interés (con signo negativo), la utilización, los beneficios y un índice de confianza; todos ellos en diferencias respecto a su nivel normal. Sus estimaciones muestran la importancia relativa de esas variables exógenas en diferentes períodos muestrales tanto para a la economía española como para la de Estados Unidos.

López Prol y Palazuelos (2016) analizan el crecimiento de la economía española entre 1994 y 2007, destacando la interrelación positiva entre la tasa de rentabilidad y la inversión a partir de un comportamiento dicotómico. Por un lado, los sectores vinculados a la burbuja inmobiliaria que se produjo en esos años, sobre todo la construcción y los servicios financieros, son los que presentan una fuerte interacción rentabilidad-inversión. Por otro lado, en los demás sectores la relación es menor en unos y no existe en otros. Analiza también los distintos comportamientos en cuanto a la tasa de rentabilidad, de manera que en unos sectores se basa en la eficiencia del capital (servicios financierosprofesionales y manufacturas de media tecnología), mientras que en la construcción y la mayoría de los restantes sectores el aumento de la rentabilidad se basa en el incremento de la cuota de los beneficios en el valor añadido, i.e. a costa del menor crecimiento de los salarios unitarios reales con respecto a la productividad laboral.

De igual forma, Alberdi (2013) señala que la expansión crediticia y el apalancamiento generaron los respectivos incrementos de la rentabilidad y la acumulación, a la vez que una inflación en los precios de los activos inmobiliarios. Del Río (2015) advierte que la relación entre beneficios e inversión de las grandes empresas se mantiene durante el período y sólo se interrumpe a partir de 2010, cuando la evolución de la inversión ya no se corresponde con el ritmo de crecimiento de los beneficios; lo cual también destaca Salas (2014).

\section{HECHOS OBSERVADOS E HIPÓTESIS SOBRE LA INVERSIÓN EN ESPAÑA}

Este apartado sintetiza un conjunto de hechos relevantes que se observan en la economía española del período 1982-2007 referidos a la dinámica de la inversión y de las variables que la literatura señala como posibles determinantes: rentabilidad, demanda o utilización de capacidad, condiciones financieras y otras que también podrían estar vinculadas a la inversión.

La elección del periodo se debe a que el objeto de estudio es el período expansivo que, con la breve excepción de la recesión de 1993, supuso la larga etapa de crecimiento e inversión (Tabla 1).

De ese modo, a partir de las propuestas teóricas presentadas en el apartado anterior y de los hechos observados, se proponen dos hipótesis que posteriormente se someten a contrastación.

1) Fuerte ritmo de crecimiento de la formación bruta de capital fijo (FBCF) que todavía fue mayor en el caso de la inversión neta que garantiza la ampliación del stock de capital. La tasa media de crecimiento de la FBCF fue del 4,9\% anual, debido principalmente a que el incremento del intervalo 
1994-2007 (6,2\%) casi duplica al registrado en 1982-1993 (Tabla 1). En términos de tasa de acumulación (FBCF/stock bruto $\mathrm{CF}$ ), el crecimiento arroja una tasa media del 1,4\% anual, de nuevo sustentada en el fortísimo dinamismo del segundo intervalo cuando creció al 2,2\% anual.

2) Según la composición técnica de las inversiones, el mayor incremento se ha concentrado en equipamiento, aunque se mantiene el predominio de la inversión en instalaciones. La FBCF en equipamiento, formada por maquinaria, instrumentos de trabajo, material de transporte, software y otros activos inmateriales, creció a un ritmo del 6,1\% anual, mientras que la realizada en instalaciones (vivienda residencial, infraestructuras y otras construcciones) lo hizo al 4,5\%

3) Según el agente inversor (véase Cárdenas, 2017), el mayor incremento ha correspondido a las administraciones públicas y a las empresas, pero también ha sido importante la inversión residencial de los hogares. La FBCF realizada por el Estado y por las empresas ha crecido por encima del 5\% anual, mientras que la de los hogares lo ha hecho casi al 4\% (Tabla 1 ).

4) Dentro del sector empresarial, la inversión ha crecido bastante más y se concentra de forma mayoritaria en las ramas creadoras de empleo, con especial relevancia en el sector de la construcción; siendo menor el crecimiento y la localización en las ramas más productivas y decepcionante el comportamiento inversor en las manufacturas.

Tabla 1

Evolución de la inversión: tasas de variación anual

\begin{tabular}{|l|c|c|c|}
\hline & $1982-07$ & $1982-93$ & $1994-07$ \\
\hline Formación bruta de capital fijo & 4,9 & 3,3 & 6,2 \\
(FBCF) & 4,5 & 3,3 & 5,5 \\
- Instalaciones & 6,1 & 3,5 & 8,4 \\
- Equipamientos & 3,9 & 0,6 & 6,8 \\
\hline Hogares & 5,8 & 9,5 & 2,7 \\
Administraciones públicas & 5,3 & 3,5 & 6,8 \\
Empresas & 8,8 & 7,6 & 9,8 \\
* Sectores creadores empleo (a) & 10,4 & 4,9 & 15,3 \\
- Construcción & 3,8 & 1,2 & 6,2 \\
(b) Sectores intensivos en capital & 2,2 & 3,9 & 0,8 \\
* Sectores manufactureros & 1,4 & 0,5 & 2,2 \\
\hline Tasa de Acumulación Buta (I/K)
\end{tabular}

Notas: a) Construcción, Hostelería, Inmobiliario sin vivienda, Educación y Sanidad privada, Servicios Profesionales, Comercio y Otros servicios. (b) Suministros básicos (agua, gas, electricidad), finanzas, Transporte e información-comunicación.

Fuente: Elaboración propia a partir de datos BBVA-IVIE.

5) Fortísimo crecimiento de la financiación a los hogares y a las empresas, sobre todo de los préstamos a largo plazo concedidos bajo garantía real, en un contexto monetario netamente expansivo. El conjunto del crédito concedido por el sector financiero a los "otros sectores residentes" (OSR) creció a casi un 7\% anual en términos reales, superando el 10\% en el segundo intervalo. Hasta 1985 el crédito al sector privado se incrementó al mismo ritmo que el PIB, después lo hizo a un ritmo mayor y desde 1996 lo hizo a una tasa media anual que triplicaba la correspondiente al PIB. La expansión crediticia ha sido más intensa en los préstamos concedidos con garantías reales, compuestos en su mayoría por hipotecas, ya que registraron un crecimiento medio de casi el $14 \%$ anual, que aún fue mayor en el segundo intervalo.

6) La expansión de la demanda interna impulsó la utilización de capacidad instalada a la vez que creó mayores necesidades de financiación exterior de la economía mientras se debilitaba el ahorro interno. Esa fortaleza interna de la demanda, complementada por la parte de importaciones destinadas a insumos y equipos productivos, impulsó la utilización de la capacidad instalada, sobre todo durante el segundo intervalo cuando se elevó desde el $72 \%$ en 1994 hasta el 82\% en el 2007 (Fuente: BDSICE).

7) Los ingresos del sector público crecieron bastante más que los del sector privado y, dentro de estos, los de las empresas aumentaron más que los de los hogares. La renta bruta disponible de los hogares se incrementó al 2,8\% a lo largo de todo el período, siendo similares los aumentos en los dos 
intervalos. Si únicamente se consideran los salarios, el crecimiento de todo el período 1982-2007 fue del 2,4\% anual, debido principalmente al fuerte aumento del número de ocupados $(2,1 \%)$, mientras que el aumento del salario unitario por ocupado fue de sólo el $0,3 \%$.

Las evidencias que revelan los hechos observados, comprobamos que las respuestas a ambos interrogantes han de tener en consideración lo siguiente:

- Que es imprescindible analizar por separado la inversión que realizan los hogares y el Estado porque, según muestran los hechos, de forma conjunta representa cerca del $45 \%$ de la inversión total.

- Que es posible que los tres grupos de factores que los estudios teóricos plantean como determinantes de la inversión de las empresas (expectativas-rentabilidad, demandautilización de capacidad y financiación) también estén presentes en las decisiones de inversión de los otros dos agentes (hogares y Estado).

- Que a la vez es previsible que sean distintas las variables específicas en las que se concretan las decisiones de invertir en viviendas (hogares), infraestructuras (Estado) y stock productivo (empresas).

\section{FUNCIONES DE INVERSIÓN DE LOS TRES AGENTES}

En consecuencia, y teniendo en cuenta las tres últimas cuestiones señaladas en el apartado anterior, cabe plantear como primera hipótesis (H.1): que las funciones de inversión de los tres agentes considerados estarán determinadas por variables referidas a las expectativas, el nivel de demanda y el acceso a financiación, pero que dichas variables pueden ser aproximadas por indicadores diferentes según cada agente.

Finalmente, y a la luz de los dos hechos anteriores, se formula como segunda hipótesis (H.2): que las actividades relacionadas con la construcción, impulsadas y sostenidas por la actuación del Estado, han tenido una importancia decisiva en el comportamiento agregado de la inversión durante todo el periodo.

\subsection{Inversión de los hogares}

La variable dependiente es la FBCF de las viviendas, medida en términos reales a precios constantes de 2005, cuya fuente es la base BBVA-IVIE. Las variables exógenas de cada grupo de factores determinantes son: i) el ahorro de los hogares (AH), como proxy de las expectativas de los hogares, ii) el empleo, medido por el número de ocupados (O), como proxy de la demanda de viviendas, y iii) los préstamos hipotecarios concedidos a los hogares $(\mathrm{CH})$ Como se expone más adelante, también se han realizado diversas pruebas con otras variables. La fuente de los datos de ahorro y préstamos es el Banco de España y los datos han sido deflactados por el IPC (INE) al considerar que es el indicador de precios que mejor se asocia a las decisiones de los hogares. Los datos cuya frecuencia es inferior al año han sido compactados mediante el cálculo de promedios si se trata de variables stock y mediante la suma si se trata de variables flujo.

Dado que estas variables presentan raíz unitaria, el modelo ha sido estimado con las variables explicativas en diferencias logarítmicas y con la variable endógena con modelo I(1), de tal forma que con esta transformación las series son estacionarias. Asimismo, se ha incluido una variable dummy en el año 1991, indicativa de que en ese año se ha producido una caída de la inversión que no puede explicarse por las variables exógenas utilizadas. Se trata de una corrección necesaria dado que para ese año se han encontrado residuos significativamente distintos (superiores a 2.5 la desviación típica). Ello hace necesario ese análisis de intervención cuya ausencia distorsionaría los posibles resultados.

Finalmente, las pruebas realizadas han mostrado que la variable de financiación con mejor ajuste es la de los préstamos hipotecarios con el adelanto de un año, lo que implica que todos los valores de la serie se han trasladado un período hacía el pasado, de tal forma que la serie para el período 1982-2007, toma los valores de 1983-2008. Desde el punto de vista económico, refleja el requisito previo de contar con financiación que acompaña a este tipo de inversión en vivienda, de modo que la expectativa de contar con financiación es previa a la adquisición de vivienda. 
El modelo finalmente estimado presenta un buen ajuste. Las variables incorporadas como explicativas superan los test de significatividad, los residuos del modelo son ruido blanco y superan los test habituales de ausencia de autocorrelación y del efecto ARCH, así como presencia de distribución gaussiana de las innovaciones.

Dado que la constante no es significativa, se excluye de la formulación del modelo, quedando la siguiente especificación1 de la estimación:

$$
\begin{aligned}
& \begin{array}{l}
(1-L) \\
+\hat{a}_{t}
\end{array} \\
& \qquad \begin{array}{l}
H \\
\end{array} \\
& (0,03) \quad 0,083 * * A^{H}+1,85 * * * O+0,05 * C^{H}\left(L^{-1}\right)-0,073 * * * D_{91} \\
& (0,163) \quad(0,026)
\end{aligned}
$$

$$
\text { Ljung-Box Q' (2)= 0,48 (0,79); Chi-cuadrado }(2)=0,321(0,85)
$$

Según los resultados, la variable de demanda (número de ocupados) es la que presenta una mayor elasticidad, seguida a distancia de la de expectativas (ahorro) y de la de financiación (préstamos hipotecarios). No obstante, hay que reiterar que no se trata de puede establecer una relación mecanicista y unicausal de los factores determinantes de la inversión, ya que, de un lado, las tres variables interactúan a lo largo del tiempo, sin que tenga sentido separar sus efectos y, de otro lado, los rangos de variación de las tres variables son muy diferentes.

También es interesante destacar otros tres resultados obtenidos durante las pruebas realizadas. En primer lugar, la introducción de una variable de ingresos (renta bruta disponible o bien salarios), en lugar del ahorro como proxy de las expectativas de los hogares, no resulta significativa ni cuando se toma como contemporánea ni cuando se hace con retardos. En segundo lugar, tampoco resulta significativa la incorporación de una variable referida a los precios de la vivienda, considerando el IPC o, con mejor criterio, el deflactor de precios de la inversión de los hogares (cuya fuente es BBVAIVIE). En tercer lugar, cuando se incorpora el tipo de interés (de los préstamos hipotecarios) como variable que expresa el coste de la financiación se obtiene que su coeficiente es negativo. Los tres resultados son relevantes porque apuntan a que el acceso a la financiación eludió las restricciones provenientes de los ingresos y de la subida de precios de las viviendas durante la burbuja inmobiliaria, apuntando el carácter especulativo de una parte de la inversión en vivienda, que habría sido realizada no por necesidades habitacionales sino por las expectativas de revalorización al calor de la burbuja, sin importar el encarecimiento de las viviendas adquiridas con el propósito de venderlas a un precio superior.

\subsection{Inversión de las Administraciones Públicas}

En primer lugar, como ya se ha expresado, es necesario precisar que la mayoría de estudios consideran que la inversión pública es autónoma (exógena), pues responde a factores discrecionales de la actuación estatal. Responde principalmente a elementos como la orientación ideológica de los gobiernos, las condiciones políticas existentes, las coyunturas del ciclo económico, la capacidad de endeudamiento y financiación de los distintos estados en los mercados de capitales y los contextos internacionales vigentes. Aunque esta idea es compartida en la presente investigación, no obstante, para homogeneizar el tratamiento con los otros dos agentes según se ha expuesto en la primera hipótesis propuesta, es interesante considerar la posibilidad de que la inversión pública obedezca a ciertas variables de referencia (aunque lógicamente no cubran toda la casuística posible). Considerando esta idea cabe hacer un planteamiento que aproxime las categorías generales, como el que sigue.

\footnotetext{
${ }^{1}$ Estimación mediante Máxima Verosimilitud exacta. Dado que los residuos no presentan ninguna estructura no es necesario incorporar elementos de media móvil o autorregresivos, por lo que la estimación es equivalente a una realizada mediante MCO.
} 
La variable dependiente es la FBCF del conjunto de las administraciones públicas (IP), medida en términos reales a precios constantes de 2005 (Fuente: BBVA-IVIE). Casi en su totalidad, en torno al $90 \%$, se trata de inversiones en infraestructuras realizadas por el gobierno central en redes viarias, ferroviarias, portuarias, aeroportuarias, hidráulicas, junto con otras construcciones realizadas por los gobiernos autonómicos y los ayuntamientos. Para establecer las variables explicativas, siguiendo la propuesta de los tres grupos de factores, cabe considerar que ha existido un programa continuado de inversión pública (Álvarez y Lagos, 2013) en todos los niveles de la Administración con el fin de subsanar el atraso relativo de las infraestructuras, nacionales y regionales y de fomentar la inversión privada (especialmente la residencial) según crecía la población.

De ese modo, dadas las características de la inversión pública, se ha optado por incorporar dos tipos de variables exógenas, una referida a los ingresos públicos y la otra a la financiación: i) los ingresos (IngP) de las administraciones (Fuente: BDREMS), y ii) las obligaciones reconocidas por el Estado (CapP) correspondientes a las operaciones de capital (Fuente: BDSICE), que se refieren a los pasivos financieros que resultan de la suma de inversiones reales y transferencias de capital de las administraciones. Se trata de la mejor variable proxy de la financiación de la inversión estatal, debido a las dificultades metodológicas e informativas que presentan otras variables. Las dos variables han sido deflactadas por el deflactor del PIB (Fuente: BDREMS) por considerar que es el indicador que mejor se asocia a las decisiones del Estado sobre inversión. De nuevo, los datos cuya frecuencia es inferior al año han sido compactados mediante el cálculo de promedios si se trata de variables stock y mediante la suma si se trata de variables flujo.

Se ha estimado un modelo ARIMAX del tipo IMA(1,1) mediante máxima verosimilitud, con esas dos variables exógenas y donde los ingresos públicos se tratan del mismo modo que con la variable de financiación en el caso anterior, i.e. se han adelantado un año. De modo que su serie corresponde al período 1983-2008. Se requiere también incorporar una dummy en el año 1982 cuyo comportamiento no se puede explicar mediante esas variables. El modelo presenta un buen ajuste, los residuos son ruido blanco y se superan los test de normalidad, ausencia de autocorrelación y heterogeneidad. La formulación estimada es:

$$
\begin{gathered}
(1-L) I^{P}=-0,001 \gamma+1,08 * * * \operatorname{Ing}^{P}+0,56 * * * \text { Cap }^{P}-0,17 * D_{82}+\hat{a}_{t}- \\
0,65 * * * \hat{a}_{t-1} \\
(0,38) \quad(0,15) \quad(0,09) \quad(0,18)
\end{gathered}
$$

$$
\text { Ljung-Box Q' }(2)=3,88(0,14) \text {; Chi-cuadrado }(2)=0,20(0,90)
$$

Según los resultados, el modo como operan los ingresos públicos sobre la inversión puede ser interpretado en cierta manera como proxy de las expectativas. A su vez, la elasticidad de los ingresos es superior a la unidad y también es notable la que corresponde a la financiación; si bien hay que volver a insistir en que la correcta interpretación de la influencia de esos coeficientes sobre la inversión necesita que se tenga en cuenta el diferente rango de variabilidad de cada variable explicativa, siendo muy superior en el caso de la financiación.

\subsection{Inversión de las empresas}

La variable dependiente es la FBCF corporativa (IC), realizada por las empresas privadas y públicas, medida en términos reales a precios constantes de 2005 y calculada como la diferencia entre la FBCF total y la correspondiente a los hogares y al Estado (Fuente: BBVA-IVIE). Las variables exógenas pertenecientes a cada grupo de factores determinantes son: i) la tasa de ganancia esperada o rentabilidad esperada (rn), calculada por la ratio beneficios/stock de capital fijo menos el tipo de interés (medido como el tipo de interés a 3 meses deflactado por el deflactor del PIB), como proxy de las expectativas de rentabilidad de las empresas (Fuentes: EUKLEMS, BBVA-IVIE y BdE); ii) el grado de utilización (u) de la capacidad productiva de la industria (Fuente: BDSICE), como proxy del comportamiento de la demanda; y iii) los créditos a empresas (CE) en términos reales utilizando el deflactor del PIB (Fuente: BdE). Las carencias de la información disponible aconsejan excluir otras formas de financiación a través de los mercados de capital (bonos y acciones). De nuevo, los datos 
cuya frecuencia es inferior al año han sido compactados mediante el cálculo de promedios si se trata de variables stock y mediante la suma si se trata de variables flujo.

Utilizando la misma metodología de Box-Jenkins con análisis de intervención, se ha estimado un modelo que sigue un proceso ARIMAX del tipo ARI $(1,1)$ con las tres variables mencionadas. Ha sido necesario incorporar una variable dummy en 1993 cuyo comportamiento no se explica con las variables consideradas. El modelo finalmente presenta un buen ajuste; las variables explicativas superan los test de significatividad, los residuos son ruido blanco y superan los test habituales de ausencia de autocorrelación y del efecto ARCH. El modelo estimado (siguiendo el procedimiento de la estimación anterior) es el siguiente:

$$
\begin{gathered}
(1+0,6(L))(1-L) I^{C}=0,04 \gamma+0,15^{* * *} r^{n}(L)+0,71 * * * u+0,26^{* *} C^{C}+ \\
(0,04) \quad(0,30) \quad(0,14) \\
+0,02 D_{83}+0,04 D_{93}-0,12 * * * D_{93}+\hat{a}_{t} \\
(0,04) \quad(0,04) \quad(0,03)
\end{gathered}
$$$$
\text { Ljung-Box Q' (2)=2,44 (0,11); Chi-cuadrado }(2)=3,68(0,15)
$$

Según los resultados, la variable de demanda (grado de utilización de la capacidad instalada en la industria) es la que presenta una mayor elasticidad, seguida de las expectativas generadas por la rentabilidad esperada y por la financiación en forma de préstamos; si bien, de nuevo, hay que considerar que, según los hechos observados, el rango de variabilidad de la primera variable es bastante menor que el de los créditos y que la tasa de rentabilidad esperada. De igual forma, el hecho de que las tres variables sean significativas implica que la interpretación del modelo no permite separar los efectos de cada una de ellas, sino que se ha de considerar que determinan de forma simultánea el comportamiento de la variable dependiente.

Igualmente, se ha realizado otra prueba con el nivel de beneficios (B) como proxy de las expectativas, tomando dicha variable con retardo de un año. En este caso, se precisa incorporar una dummy correspondiente al año 1987 y el modelo obtenido presenta un ajuste incluso más favorable que el anterior, en el que tanto los beneficios esperados como el grado de utilización de la capacidad presentan coeficientes de elasticidad bastante elevados.

Los resultados de la estimación (en las mismas condiciones que la anterior) son los siguientes:

$$
\begin{aligned}
& \begin{array}{l}
(1+0,39(L))(1-L) I^{C}= \\
+\hat{a}_{t}
\end{array} \\
& (0,23) \quad(0,32) \quad(0,14) \quad(0,04)
\end{aligned}
$$

$$
\text { Ljung-Box Q' (2) = 1.96(0.16); Chi-cuadrado (2) = } 1.52(0.46)
$$

Desde una interpretación convencional, que es bastante habitual, esos datos mostrarían la interacción que existe entre el acelerador de la inversión y el grado de monopolio a través de la inversión, de modo que los beneficios esperados estimulan el incremento de utilización de la capacidad, y la mayor utilización generaría mayores beneficios. El tercer término, la financiación, presenta un con menor coeficiente de elasticidad, pero su rango de variabilidad es notablemente mayor que el de las otras dos variables y su significatividad muestra que también es una variable relevante.

Por tanto, finalmente, con una $\mathrm{u}$ otra variable que exprese las expectativas de rentabilidad, ambos modelos permiten formular una interpretación consistente acerca de la influencia interactiva de las tres variables consideradas en el comportamiento de la inversión realizada por las empresas.

También es relevante destacar que se obtienen resultados similares cuando la variable dependiente es la tasa de acumulación (I/KC), esto es, el cociente entre la FBCF y el stock de capital bruto, en este caso ambas referidas a las empresas. Como se ha señalado al final del subapartado 2.1, esa 
especificación de la tasa de acumulación es la formulación habitual con la que se expresa la dinámica de acumulación de una economía capitalista. En este caso la estimación de un modelo ARI $(1,1)$, en las condiciones anteriores (Máxima Verosimilitud y las variables explicativas en diferencias logarítmicas) y con tres variables dummy, supera los test residuales habituales y puede considerarse validado. De tal forma que arroja los siguientes coeficientes:

$$
\begin{aligned}
& (1+0,49(L))(1-L)(I / K)^{C}=0,15 * * * r^{n}(L)+0,66^{* *} u+0,34 * * * C^{C} \\
& +(0,04) \quad(0,26) \quad(0,12) \\
& +0,09 * * * D_{8}+0,086^{* * *} D_{87}-0,11 * * * D_{93}+\hat{a}_{t} \\
& (0,03) \quad(0,35) \quad(0,027)
\end{aligned}
$$

Ljung-Box Q' (2)=3,88 (0,14); Chi-cuadrado $(2)=0,20(0,90)$

Se aprecia la semejanza que mantiene este modelo con los dos anteriores. La tasa de acumulación del sector corporativo presenta una relación positiva con la rentabilidad, el grado de utilización y la financiación mediante préstamos como los créditos a sociedades. Es destacable que en este caso los coeficientes de las variables se encuentran en un rango de variación menor, mientras que las dummy muestran incrementos de la tasa de acumulación en los años 1986 y 1987, así como una caída en 1993, que no pueden ser explicados por las variables exógenas.

\section{FUNCIÓN DE INVERSIÓN AGREGADA DE LA ECONOMÍA}

Para abordar la segunda hipótesis se utiliza una metodología similar a la empleada en el apartado anterior, mediante un modelo que incorpora el papel decisivo que juegan las actividades relacionadas con la construcción. La variable dependiente es la FBCF del conjunto de la economía (IT), medida en términos reales a precios constantes de 2005 (Fuente: BBVA-IVIE). En un segundo modelo se considera como variable dependiente la tasa de acumulación $(\mathrm{I} / \mathrm{K})$, medida como la inversión bruta sobre el stock de capital bruto.

Las variables exógenas combinan las referidas al sector de construcción con otras del conjunto de la economía: i) la FBCF de "actividades vinculadas con la construcción" (AVC) que consiste en la inversión (IAVC)conjunta de las AA.PP (infraestructuras) los hogares (vivienda) y la propia del sector empresarial de construcción (BBVA-IVIE); ii) como variable de expectativas se incluyen, primero, la tasa de ganancia esperada del sector empresarial de la construcción ( $\mathrm{rCT}$ ), calculada por la ratio beneficios/stock de capital fijo del sector menos el tipo de interés real (medido como el tipo de interés a 3 meses deflactado por el deflactor del PIB), como proxy de las expectativas de rentabilidad del sector (calculada a partir de datos EUKLEMS y BBVA-IVIE); iii) el grado de utilización de la capacidad instalada en la industria, como proxy de la demanda agregada (Fuente: BDSICE); y d) los créditos a los sectores residentes $(\mathrm{F})$, i.e. la financiación vía préstamos a los agentes internos no financieros (Fuente: BdE). Los datos de la variable dependiente y de las variables exógenas están expresados en términos reales, habiéndose utilizando el deflactor del PIB. Los datos cuya frecuencia es inferior al año han sido compactados mediante el cálculo de promedios si se trata de variables stock y mediante la suma si se trata de variables flujo.

Aplicando la metodología de Box-Jenkins con análisis de intervención se obtiene que el primer modelo estimado sigue un proceso ARIMAX del tipo ARI $(1,1)$. La tasa de ganancia incorpora un retardo de un año, mientras que las demás variables se expresan en datos contemporáneos. El correlograma de los residuos indica que son ruido blanco con media cero y varianza constante, dado que no se observa ninguna estructura modelizable, se han realizado los contrastes residuales habituales, comprobando que no existen problemas de autocorrelación, ni de heterocedasticidad condicional o efectos ARCH, y que siguen una distribución normal.

Por tanto, el modelo que presenta un buen, ajuste se formula como: 


$$
\begin{aligned}
& (1+0,73(L))(1-L) I^{T}=0,10^{* * *}{ }^{C T}(L)+0,41^{* *} u+0,50 * * F-0,06^{* * *} \\
& D_{84}+\hat{a}_{t} \\
& (0,05) \quad(0,19) \quad(0,12) \quad(0,02)
\end{aligned}
$$

$$
\text { Ljung-Box Q' (2) =0,77(0,37); Chi-cuadrado }(2)=0,95(0,62)
$$

Los resultados de la estimación son claros, todos los coeficientes son significativos y con los signos esperados. En primer lugar, la rentabilidad esperada en el sector de la construcción ha tenido un papel relevante, lo que avala el papel de las expectativas en la evolución de la inversión. De igual forma, la utilización refleja el efecto acelerador inducido desde la demanda y la evolución del crédito las favorables condiciones financieras en las que se desarrolló la inversión. Además, se ha añadido una variable dummy para el año 1984 para mejorar el ajuste del modelo, ya que hay una caída de la inversión total que no puede explicarse por las variables exógenas en ese año.

En segundo lugar, para valorar la incidencia del agregado AVC sobre el conjunto de la economía, se incluye como variable explicativa en el modelo anterior. El modelo estimado sigue un proceso ARIMAX del tipo ARI $(1,1)$. Tanto la inversión de las AVC como la tasa de ganancia incorporan un retardo de un año, mientras que las demás variables se expresan en datos contemporáneos; además se incluye una variable dummy para el año 1984. El análisis de los residuos muestra que siguen un proceso de ruido blanco, superando los test habituales. Además, debido a que una de las variables explicativas forma parte de la dependiente se han realizado varias pruebas en los test de ausencia de autocorrelación, siendo superados en todas las modalidades probadas (desde los 2 a los 5 retardos).

$$
\begin{aligned}
& (1+0,7(L))(1-L) I^{T}=0,25 * * I^{A V C}(L)+0,13 * * * r^{C T}(L)+0,38 * * u+ \\
& 0,35 * * * F-0,05 * * * D_{84}+\hat{a}_{t} \\
& (0,05) \quad(0,11) \quad(0,005) \quad(0,17) \quad(0,13)
\end{aligned}
$$

$$
\text { Ljung-Box } Q^{\prime}(2)=0.24(0.62) \text {; Chi-cuadrado }(2)=0.32(0.85)
$$

Por tanto, la principal diferencia con el modelo anterior es la inclusión de la inversión de las AVC con un retardo, que su coeficiente sea positivo y significativo con un retardo, lo cual resalta el liderazgo de la inversión de los tres agentes que está relacionada con la construcción, cuya expectativa favorable de crecimiento parece haber ejercido como factor impulsor de la inversión del total. Su elasticidad es menor que uno porque se trata de es un agregado más volátil, pero lo relevante es que tiene efectos en el conjunto. Las otras variables muestran coeficientes similares a los del modelo anterior, siendo todos los signos los esperados.

En definitiva, las estimaciones propuestas para el conjunto de la economía permiten observar que las tres variables del esquema propuesto (expectativas, demanda y financiación) han jugado papeles relevantes en la evolución de la inversión. Asimismo, la inclusión de la inversión debido a la importancia de las AVC, se ha considerado su inversión como la de un sector líder, comprobándose que efectivamente su crecimiento ha tenido una muestra su efecto positivo sobre el conjunto de la inversión.

\section{CONCLUSIONES}

El objetivo de este trabajo es investigar el comportamiento de la inversión de la economía española durante el período 1982-2007, limitando la investigación a este período con el objetivo de explicar por qué registró altas tasas de crecimiento y por qué se concentró principalmente en los sectores creadores de empleo y de forma particular en la construcción. Las formulaciones teóricas afines al núcleo de proposiciones teóricas proponen tres tipos de factores explicativos de la inversión: las expectativas de rentabilidad, el comportamiento de la demanda agregada y el acceso a financiación, si bien la mayoría de los estudios teóricos y empíricos están referidos al comportamiento de las empresas. Sin embargo, 
los hechos observados en la economía española muestran que la inversión realizada por el Estado y los hogares representa cerca del $45 \%$ de la inversión total y, además, la secuencia seguida por esas inversiones, concentradas en infraestructuras y en viviendas, sugiere que existen vínculos importantes entre la inversión empresarial, en la que destaca el sector de la construcción, y esas inversiones estatales y domésticas.

Según la primera hipótesis, cabe encontrar funciones de inversión para cada uno de los tres agentes que incorporan variables referidas a las expectativas, el nivel de demanda y el acceso a financiación; pero, a la vez, hay diferencias en los indicadores específicos que aproximan los comportamientos respectivos de cada agente. Según la segunda, cabe encontrar una función de inversión para el conjunto de la economía que incorpora el papel decisivo de las actividades relacionadas con la construcción (condicionadas por la actuación del Estado), a la vez que las variables incluidas en las categorías teóricas de rentabilidad, demanda y de financiación.

Los resultados que proporcionan los ejercicios de estimación realizados, para cada uno de los agentes y a nivel agregado, aportan tres conclusiones principales.

Primera: Se confirma la primera hipótesis, pero con un matiz en el caso de la inversión del Estado. La financiación es una variable destacada en las funciones de inversión de los tres agentes, referida a los préstamos hipotecarios (hogares), los créditos (empresas) y los pasivos financieros por inversiones reales y transferencias de capital (AAPP). En los tres casos las variables utilizadas se refieren a los préstamos como proxy del acceso a financiación, sin necesidad de precisar si procede de ahorro interno de la economía o de fuentes internacionales, y sabiendo que las empresas y el Estado cuentan también con la emisión de títulos de deuda y en el caso de las empresas con la emisión de nuevas acciones para ampliar las posibilidades de financiar sus inversiones. Igualmente, en los tres agentes influye una variable de expectativas, referida al ahorro (hogares), la tasa de ganancia esperada (empresas) y los ingresos públicos (AAPP). En dos agentes se incorpora una variable de demanda, el empleo (hogares) y la utilización de capacidad (empresas). El matiz corresponde a la inversión estatal, ya que por su propia naturaleza discrecional e ideológico-política, las decisiones inversoras de las administraciones difícilmente pueden asociarse a expectativas uniformes o responden a demandas específicas.

Segunda: Se confirma la segunda hipótesis conforme a dos modelos relevantes. El primero explica la dinámica seguida por la inversión agregada de la economía mediante las expectativas de la tasa de beneficio en el sector de la construcción, la utilización de capacidad instalada y la financiación recibida por los tres agentes. El segundo incorpora una cuarta variable a las tres anteriores: las expectativas de inversión conjunta en las actividades vinculadas a la construcción, como muestra del proceso autorreproductivo generado a partir de la interrelación existente entre las decisiones de inversión de las AAPP (infraestructuras), los hogares (viviendas) y el propio sector empresarial dedicado a la construcción.

Por tanto, los resultados obtenidos confirman los que ya habían propuesto otros autores sobre la importancia de la inversión en viviendas (Álvarez y Cabrero, 2010), el efecto del crédito en la inversión residencial (Arestis y González, 2016) y en bienes de equipo (Posada, Urtasun y González, 2014). Pero al mismo tiempo, esos resultados aportan una explicación más concreta sobre la inversión realizada por los distintos agentes y una explicación relevante de la inversión agregada de la economía basada en la interrelación de esos comportamientos por agentes.

Tercera: Las dos preguntas acerca del ritmo de inversión y su concentración sectorial, tienen una misma respuesta articulada. Dadas las características de la estructura productiva y del tipo de concentración empresarial existentes, la posibilidad de que la economía española registrase fuertes incrementos de la inversión era a través de su localización mayoritaria en los sectores creadores de empleo, y de forma particular en el sector de construcción.

Finalmente, los resultados obtenidos y las conclusiones presentadas reclaman nuevas investigaciones que fortalezcan su consistencia explicativa y que aporten nuevos conocimientos sobre el comportamiento de la inversión en España. Una línea consiste en incorporar otras posibles variables proxy de las tres variables (expectativas, demanda y financiación) y otras posibles variables que también puedan explicar esa dinámica de inversión. 


\section{AGRADECIMIENTOS}

Este trabajo se ha beneficiado de los valiosos comentarios de mis directores de Tesis, Rafael Fernández y Enrique Palazuelos (UCM), y de dos evaluadores anónimos. Asimismo, una versión previa de este artículo fue presentada en el XXXI Congreso Internacional de Economía Aplicada y en un seminario organizado por el Instituto de Investigaciones Económicas de la Universidad Nacional Autónoma de México, por lo que hago extensible mi agradecimiento a los organizadores y participantes en aquellas sesiones. En cualquier caso, cualquier error es de mi exclusiva responsabilidad

\section{REFERENCIAS BIBLIOGRÁFICAS}

ALBERDI, A. (2013). El sistema bancario y la financiación del crecimiento de la economía vasca. Ekonomiaz. 84(03), 236-273.

ALLSOPP, C., \& GLYN, A. (1999). The assessment real interest rates. Oxford Review of Economic Policy, 15(2), 1-16.

ALONSO, C. \& BENTOLILA, S. (1994). Investment and Q in Spanish manufacturing firms. Oxford Bulletin of Economics and Statistics, 56(1), 49-65.

ARESTIS, P., \& GONZÁLEZ, A. R. (2016). House Prices and Current Account Imbalances in OECD Countries. International Journal of Finance \& Economics, 21(1), 58-74.

ARESTIS, P., GONZÁLEZ, A. R., \& DEJUÁN, Ó. (2012). Private productive investment in Spain and the United States. Análise Econômica, 30(58).

ARRIOLA, J. (1999). Capacidad instalada y producción potencial en la industria vasca. Ekonomiaz, 43(01), 210231.

AZOFRA, V. \& LÓPEZ, F. J. (1997). Modelos de inversión en activo fijo: determinantes y propuestas. Anales de estudios económicos y empresariales, 12(1), 7-26.

BLANCH, J. (1988). Inversión y teoría Q en la economía española. Cuadernos de Economía, 16(47), 367-388.

BLECKER, R. A. (2015). Wage-led versus profit-led demand regimes: The long and the short of it. Eastern Economic Association, New York, NY.

CÁRDENAS, L. (2017). Hechos estilizados del patrón de inversión en la economía española (19812013). Revista Galega de Economía, 26(2), 5-16.

CÁRDENAS, L. (2018). Notas sobre la teoría kaleckiana de la inversión. Cuadernos de Economía, 41(115), 119129.

COURVISANOS, J. (2012). Cycles, Crises, Innovation. Path to Sustainable Development A KaleckianSchumpeterian Synthesis. Edward Elgar Publishing

CROTTY, J. R. (1992). Neoclassical and Keynesian approaches to the theory of investment. Journal of Post Keynesian Economics, 14(4), 483-496.

DEL RíO, A. (2015). La financiarización a debate: la desvinculación entre beneficios e inversión en el caso español. Investigación Económica, 74(291), 53-77.

EPSTEIN, G. (1994). A political economy model of comparative central banking. In New Perspectives in Monetary Economics Explorations in the Tradition of Hyman Minsky., ed. G. and R.N. Pollin Dymsky. Ann Harbor: University of Michigan Press.

ESPASA, A., \& SENRA, E. (1993). Consideraciones sobre la función de inversión en España. Documento de Trabajo, UC3M

ESTEVE, V. \& TAMARIT, C. R. (1994). Inversión agregada, Q de Tobin y capacidad instalada: una nota empírica. Economía Industrial, 298), 145-150.

ESTRADA, Á., DE CASTRO, F., HERNANDO, I. \& VALLÉS, J. (1997). La inversión en España: un análisis desde el lado de la oferta. Madrid: Servicio de Estudios del Banco de España.

FAZZARI, S. M. \& MOTT, T. L. (1987). The Investment Theories of Kalecki and Keynes: An Empirical Study of Firm Data, 1970-1982. Journal of Post Keynesian Economics, 9, 171-187.

FELIPE, J. (2002). 11. Unemployment and profitability: the case of Spain. In: Davidson, P. (ed.) A Post Keynesian Perspective on 21st Century Economic Problems. Cheltenham: Edgar Elgar Publishing.

GLYN, A. (1997). Does aggregate profitability really matter. Cambridge Journal of Economics, 21(5), 593-619.

HEIN, E. (2014). Distribution and growth after Keynes: A Post-Keynesian guide. Edward Elgar Publishing.

KALECKI, M. (1937). The principle of increasing risk. Economica, 4(16), 440-447.

KEYNES, J. M. (1936[1956]). Teoría general de la ocupación, el interés y el dinero. México D.F.: Fondo de Cultura Económica, FCE.

LÓPEZ-PROL, J., \& PALAZUELOS, E. (2016). La relación entre beneficios e inversión en el crecimiento económico de España 1994-2007. Problemas del Desarrollo. 46(185), 111-136

MORAL, J. (2008). Restricciones al endeudamiento: una propuesta de análisis a partir del diferencial entre el ratio de rentabilidad del activo y el coste de la deuda. Cuadernos de Economía, 31(85), 107-127.

MOTT, T. (2009). Kalecki's principle of increasing risk and Keynesian economics. Routledge. 
MUÑOZ DE BUSTILLO, R. \& FERNÁNDEZ MACÍAS, E. (2007). Producción y Tiempo. Utilización de capacidad instalada en las empresas españolas. Estudios de economía aplicada, 25(1), 387-418.

PALAZUELOS, E. \& FERNÁNDEZ, R. (2007). La tasa de beneficio en la dinámica económica de los países europeos, 1984-2003. Estudios de Economía Aplicada, 25, 821-26.

POSADA, D., URTASUN, A. \& GONZÁLEZ, J. (2014). Un análisis del comportamiento reciente de la inversión en equipo y de sus determinantes. Boletín Económico. Banco de España, 2014(6), 41-51.

RICHARDSON, C., \& ROMILLY, P. (2008). Investment functions and the profitability gap. Journal of Post Keynesian Economics, 31(1), 35-56.

SALAS, V. (2014). Relación entre beneficios empresariales y la recuperación de la economía española. Cuadernos de Información económica, (242), 11-20.

SEGUINO, S. (1999). The investment function revisited disciplining capital in South Korea. Journal of Post Keynesian Economics, 22(2), 313-338.

SHERMAN, H. J. (2014). The Business Cycle: Growth and Crisis under Capitalism. Princeton: Princeton University Press.

STEINDL, J. (1952 [1976]). Maturity and stagnation in American capitalism. New York: Monthly Review Press.

WEISSKOPF, T. E. (1979). Marxian crisis theory and the rate of profit in the postwar U.S. economy. Cambridge Journal of Economics, 3(4), 341-378. 